\title{
The Analgesic Effect of Transcutaneous Electrical Acupoint Stimulation on Labor: A Randomized Control Study
}

This article was published in the following Dove Press journal: International Journal of General Medicine

\author{
Wei-Hong $Q i^{1, *}$ \\ Wei-Juan Miao ${ }^{2, *}$ \\ Yu-Zhi Ji' \\ Chao Li' \\ Jun-Huan Wang' \\ 'Department of Obstetrics, The \\ Affiliated Hospital of Qingdao University, \\ Qingdao, 266000, People's Republic of \\ China; ${ }^{2}$ Department of Obstetrics, Ri- \\ Zhao People's Hospital, Ri Zhao, 276800, \\ People's Republic of China
}

*These authors contributed equally to this work
Correspondence: Wei-Hong Qi Department of Obstetrics, Affiliated Hospital of Qingdao University, No. 59 of Haier Road, LaoShan District, Qingdao, Shandong, 266000, People's Republic of China

Tel $+86532-82913296$

Fax +86 532-82913018

Email qiweihong45@163.com
Objective: This study aimed to investigate the analgesic effect of transcutaneous electrical acupoint stimulation (TEAS) on labor.

Methods: Primiparas with single birth and head presentation were enrolled in this study and randomly divided into three groups: TEAS group $(\mathrm{n}=76)$, patient-controlled epidural analgesia (PCEA) group $(n=75)$, and control group without any analgesic measures $(n=78)$.

Results: Compared with the control group, the visual analog scores of the TEAS group and the PCEA group at each time point decreased $(\mathrm{P}<0.01)$. The decrease was greater in the PCEA group than that in the TEAS group $(\mathrm{P}<0.01)$. At 120 minutes after analgesia, there were significant differences in plasma $\beta$-endorphin content between the TEAS group, PCEA group, and control group $(\mathrm{P}<0.01)$. The difference between the PCEA group and the control group was statistically significant $(\mathrm{P}<0.01)$. Among the parturients having a vaginal delivery, the duration of the first stage of labor was significantly shorter in the TEAS group and control group than in the PCEA group $(\mathrm{P}<0.01)$. The duration of the second stage of labor was significantly shorter in the TEAS group than in the PCEA group $(\mathrm{P}<$ 0.01). Oxytocin usage rate during labor was significantly lower in the TEAS group and control group than in the PCEA group $(\mathrm{P}<0.01)$, and adverse reactions were significantly fewer in the TEAS group and control group than in the PCEA group $(\mathrm{P}<0.01)$.

Conclusion: The duration of the first and second stage of labor is significantly shorter in the TEAS group than in the PCEA group. TEAS does not increase the use rate of oxytocin or the rate of cesarean section and will not bring about obvious maternal or fetal adverse reactions. Keywords: labor analgesia, transcutaneous electrical acupoint stimulation, patientcontrolled epidural analgesia, visual analog score of pain, $\beta$-endorphin

\section{Introduction}

Pain in childbirth may exert adverse effects on mothers and children. ${ }^{1}$ Labor pain is a powerful respiratory stimulus. Hyperventilation during uterine contractions may cause severe respiratory alkalosis and a leftward shift of the maternal oxygenated hemoglobin dissociation curve, leading to fetal hypoxemia. The pain and stress of childbirth can activate the sympathetic nervous system, resulting in increased plasma catecholamine concentration, cardiac output, and blood pressure. The increase in catecholamine can also lead to a decrease in uterine blood flow. Severe labor pain can also cause serious psychological trauma, leading to an increased risk of postpartum depression. ${ }^{1}$

The current methods of labor analgesia are divided into drug analgesia and nondrug analgesia. At present, the main methods used for drug-induced labor analgesia 
include inhalation analgesia, sedative drugs, local anesthetics, and general opioids. ${ }^{2}$ In recent years, the application of a lumbar epidural block for continuous analgesia has been increasing. Its clinical analgesic effect is good, but it also has deficiencies. These include invasive infiltration, lower limb motor block, fever, urinary retention, prolonged labor, increased use of oxytocin, increased instrumental delivery rate, and the potential adverse effects of narcotic drugs in the short-term and long-term future. ${ }^{3}$ Modern labor analgesia should consider not only the analgesic effect but also the safety of mother and baby. Transcutaneous electrical nerve stimulation (TENS) is a non-invasive analgesic therapy that activates peripheral nerve fibers with electrical pulses. It has been applied to many types of clinical pain relief, such as acute trauma, postoperative analgesia, and chronic back pain. ${ }^{4}$ Transcutaneous electrical acupoint stimulation (TEAS) is a new physical analgesia method based on TENS combined with traditional Chinese medicine, meridian science, and modern electronic technology, through low-frequency pulse current stimulation to related acupoints. It dredges meridians, promotes qi and blood circulation, regulates Yin and Yang, and relieves labor pain. It has the advantages of being non-invasive, economical, and easy to operate. It is not a drug and has no obvious adverse reactions in either mother or infant. In recent years, it has been used in obstetric labor analgesia. ${ }^{5}$ In this study, we used a neuromuscular stimulator (Sunray, Guangzhou, China) to stimulate Hegu, Neiguan, Jiaji, and Cili with a lowfrequency current in order to promote qi, activate blood circulation, dredge meridians, and relieve labor pain, to explore the analgesic mechanism of TEAS and its role in labor analgesia.

\section{Materials and Methods}

\section{Materials}

\section{Subjects}

\section{Selection and Grouping of Subjects}

A total of 234 primiparas with single head positions who gave birth from January 2019 to January 2020 in our hospital were enrolled in this study and were randomly divided into three groups (in each group $\mathrm{n}=78$ ). The statistical software SPSS 22.0 was used to input the estimated sample size, generate the serial number, make the random card, put the envelopes coded in order, impervious to light and sealed, and distribute the envelopes according to the order of entering the group, and divide the selected women into 76-cases in TEAS and 75-cases in the epidural group and 78-cases in the control group that have not taken analgesic measures.

In the TEAS group, two patients shifted to an epidural block halfway because they were not satisfied with the analgesic effect of TEAS, one patient failed to complete the analgesic treatment, and the visual analog scores (VAS) of each period was withdrawn from the study due to precipitate labor. In the PCEA group, two patients failed to complete the analgesic treatment, and the VAS of each period was withdrawn from the study due to precipitate labor. Consequently, 75 cases in the TEAS group, 76 cases in the PCEA group, and 78 cases in the control group were included. The differences in age and gestational age among the three groups were not statistically significant $(\mathrm{P}>0.05)$. Hence, the three groups were comparable. This study was approved by ethics Committee of Affiliated Hospital of Qingdao University (approval No.: QYFYWZLL25584).

\section{Inclusion Criteria}

1) Primiparas with single birth and head presentation; 2) 37-41 weeks of pregnancy; 3) primiparas without cephalopelvic disproportion, intending a vaginal delivery, and without contraindications of vaginal delivery. 4) Regular contractions, the opening of the cervix was $>3 \mathrm{~cm}$ wide. 5) Puerpera and their family members agreed to implement labor analgesia and sign informed consent.

\section{Exclusion Criteria}

1) Skin damaged in the application area of the electrode piece or puncture area of PCEA. 2) Severe internal and external complications, or obstetric complications such as hypertension, diabetes, heart disease, or trauma. 3) Other analgesic measures or drugs have been used before analgesia. 4) Precipitate labor or family history of precipitate labor.

\section{Diagnostic Criteria}

The criteria were based on Obstetrics and Gynecology, ${ }^{6}$ 9th Edition, edited by Xie Xing, People's Health Publishing House.

The first stage of labor: the time from the beginning of regular contractions to the cervix fully opening.

The second stage of labor: the time from when the cervix was fully opened until the delivery of the fetus.

The third stage of labor: the time from the delivery of the fetus to the delivery of the placenta. 
Visual analog score (VAS): The basic method is as follows: a numerical scale about $10 \mathrm{~cm}$ long is used, marked with 10 scales on one side, with 0 and 10 point ends at both ends. A VAS of 0 points: no pain; $\leq 3$ points: slight pain, tolerable; $4-6$ points: the patient has pain that affects sleep, but can still endure it; $7-10$ points: the patient is gradually developing intense pain, which is unbearable, affecting appetite and sleep. The VAS was recorded immediately before analgesia, at 30 minutes, 60 minutes, and 120 minutes after analgesia. A decrease in the VAS by $\geq 3$ points was considered as effective analgesia.

Apgar score of the newborn: This consists of five physical signs: heart rate, respiration, muscle tension, laryngeal reflex, and skin color, with a full score of 2 points for each item.

\section{Main Instruments and Equipment}

Fetal monitor, low-frequency nerve and muscle stimulator, disposable electronic controlled analgesia pump, disposable anesthesia puncture bag, Sunrise enzyme microplate reader, high-speed desktop centrifuge, electric constant temperature water temperature box, medical lowtemperature preservation box, ordinary refrigerator, ultrapure water equipment.

\section{Main Drugs}

Ropivacaine hydrochloride injection, sufentanil citrate injection, lidocaine hydrochloride injection.

\section{Main Reagents}

Human $\beta$-endorphin ( $\beta$-EP), Hepeng Biological (Shanghai) Co., Ltd.

\section{Experimental Methods TEAS Method}

As shown in Supplementary Figure 1, a low-frequency nerve and muscle stimulator was used in the TEAS group. After the uterine orifice opened to $3 \mathrm{~cm}$, the patient was placed in the standing position or lateral position, and the application area was disinfected with $75 \%$ alcohol. Six electrodes were applied to Hegu (between the first and second phalanges of the hand), Neiguan ( 2 cun above the transverse line of the palmar wrist of the forearm, between the palmaris longus tendon and the flexor carpi radialis tendon), Jiaji (between T10-L1 of the spine, 0.5 cun beside the posterior midline), and Cili (between the posterior superior iliac spine and the posterior median line, opposite the second sacral posterior foramen). With the spine as the longitudinal axis, and gluteal fissure as the top, a horizontal line was drawn, and the first group of electrodes was placed for the stimulation of Ciliao acupoints. The second group of electrodes was placed at the horizontal line for the stimulation of Jiaji acupoints. At the same time, the wireless fetal heart rate monitoring probe (fetal heart rate and uterine contraction) was placed on the pregnant women's abdomens for fetal heart rate monitoring. The frequency was one $2 \mathrm{~Hz} / 100 \mathrm{~Hz}$ dilatational wave alternation per three seconds, and the current intensity was $15-50$ $\mathrm{mA}$. There are two modes. One is to slowly increase the output of current analgesia intensity manually and adjust the analgesic intensity according to the degree of pain. The other is the automatic adjustment function in which the output of analgesia intensity automatically adjusts with the strength of uterine pressure on the basic intensity value $(15 \mathrm{~mA})$. The progress of labor was observed, the fetal heart rate, uterine contraction, blood pressure, heart rate, and VAS before and after analgesia were recorded until the opening of the uterine orifice.

\section{PCEA Treatment}

The PCEA group was given epidural anesthesia. Puerperants were placed in the left-lateral position. Venous access was established using an 18-indwelling needle; Fetal heart rate and uterine contraction were monitored by fetal heart rate monitoring. Heart rate, blood pressure, and blood oxygen saturation were monitored by electrocardiogram (ECG) monitoring. The puncture point was between L2 and L3. After confirming the puncture was correct, a $5 \mathrm{~cm}$ epidural catheter was inserted into the cephalic side. It was then checked to ensure that the catheter was in the epidural space and the anesthesia block plane was below T10. First, $5 \mathrm{~mL}$ of $1 \%$ lidocaine was given, then observation was conducted for five minutes to determine whether there was a subarachnoid block or anesthetic toxicity. After excluding abnormality by observation, $8 \mathrm{~mL}$ of $0.125 \%$ ropivacaine and $0.5 \mu \mathrm{g} /$ $\mathrm{mL}$ sufentanil was injected. After the pain was relieved, the analgesia pump was connected, and $100 \mathrm{~mL}$ of $0.125 \%$ ropivacaine and $0.5 \mu \mathrm{g} / \mathrm{mL}$ sufentanil was given. The input rate was set to $10 \mathrm{~mL} / \mathrm{h}$, and the added dose was $5 \mathrm{~mL}$. The added dose interval was 15 minutes. An anesthesiologist was responsible for the whole operation process and analgesia. The progress of labor was observed, the fetal heart rate, uterine contraction, blood pressure, heart rate, and VAS before and after analgesia were recorded until the opening of the uterine orifice. 


\section{Observation of the Labor Process in the Control Group}

In the control group, the puerpera entered the delivery room for labor when the uterine orifice was $3 \mathrm{~cm}$ open. No drug or non-drug analgesia measures were taken. The progress of labor was observed. The fetal heart rate, uterine contraction, blood pressure, heart rate, and VAS before and after analgesia (before and after entering the delivery room) were recorded until the opening of the uterine orifice.

At the same time, the plasma $\beta$-EP content at 120 minutes after analgesia, the length of three stages of labor, oxytocin use rate, adverse reaction rate, conversion rate to cesarean section, postpartum hemorrhage volume, and neonatal Apgar scores were observed (detected) and recorded.

\section{Sample Acquisition}

Peripheral venous blood was collected immediately before and at 120 minutes after analgesia and centrifuged for 20 minutes (3000 rpm). The supernatant was frozen and stored in a $-20^{\circ} \mathrm{C}$ ultra-low temperature refrigerator for testing. The content of serum $\beta$-EP was measured by radioimmunoassay.

\section{Sample Detection}

Detection was carried out according to the kit's operation standards.

Determination of results: The standard curve was drawn. In the excel worksheet, with the standard concentration as abscissa and optic density (OD) value as ordinate, the linear regression curve was drawn, then the concentration value of each sample was calculated according to the curve equation.

\section{Statistics Analysis}

Statistical analysis was conducted using statistical software SPSS22.0. The VAS were compared using repeatedmeasures analysis of variance. Student-Newman-Keuls $\mathrm{Q}$ tests were used to compare the difference of $\beta$-EP content before and after analgesia between groups. Count data were compared between groups using the $\mathrm{X}^{2}$ test. Measurement data were expressed as mean \pm standard deviation ( $\mathrm{X} \pm \mathrm{SD}$ ), and compared between groups using F-tests. $\mathrm{P}<0.05$ was considered statistically significant.

\section{Results}

\section{General Situations of Puerperants}

In the TEAS group, the age of these patients ranged from $21-35$ years, with an average age of $27.6 \pm 2.8$ years. The gestational days of these patients ranged from 263-287 days,
Table I Comparison of General Situations Among Three Groups of Puerperant

\begin{tabular}{|l|l|c|c|}
\hline Groups & Cases & Age (Year) & $\begin{array}{c}\text { Number of Days of Pregnancy } \\
\text { (Day) }\end{array}$ \\
\hline TEAS group & 76 & $27.6 \pm 2.8$ & $279.1 \pm 6.4$ \\
PCEA group & 75 & $27.1 \pm 3.0$ & $277.2 \pm 7.7$ \\
Control group & 78 & $27.0 \pm 2.6$ & $278.8 \pm 6.7$ \\
F & & 1.01 & 1.630 \\
$P$ & & 0.3673 & 0.198 \\
\hline
\end{tabular}

with an average gestational length of $279.1 \pm 6.4$ days. In the PCEA group, the age of these patients ranged from 20-34 years, with an average age of $27.1 \pm 3.0$ years. The gestational days of these patients ranged from 260-287 days, with an average gestational length of $277.2 \pm 7.7$ days. In the control group, the age of these patients ranged from 21-33 years, with an average age of $27.0 \pm 2.6$ years. The gestational days of these patients ranged from 260-287 days, with an average gestational length of $278.8 \pm 6.7$ days. There were no significant differences in age and gestational length among the groups $(\mathrm{P}>0.05, \mathrm{P}>0.05$, Table 1$)$.

\section{Comparison of VAS Before and at Each Time Point After Analgesia}

Before analgesia, VAS of the three groups were normally distributed and the difference among the three groups was not statistically significant $(\mathrm{P}>0.05$, Figure 1$)$. After the implementation of analgesia, VAS analysis of the groups revealed that there were significant differences among different analgesia methods ( $\mathrm{F}$ group $=331.261, \mathrm{P}=0.000$ ). The VAS was significantly lower in the TEAS group and PCEA group than in the control group $(\mathrm{P}=0.000 ; \mathrm{P}=0.000)$. Compared with the TEAS group, the PCEA group had a larger decrease $(\mathrm{P}=0.000)$. Comparison of analgesia at different time points revealed significant changes in the TEAS group and PCEA group at 60 minutes after analgesia ( 0 minutes vs 30 minutes, $\mathrm{F}=922.801, \mathrm{P}=0.000 ; 30$ minutes vs 60 minutes, $\mathrm{F}=561.801$, $\mathrm{P}=0.000$ ). The VAS of the TEAS group and PCEA group changed slowly at 60-120 minutes after analgesia (60 minutes vs 120 minutes, $\mathrm{F}=2.769, \mathrm{P}=0.097)$. However, it maintained a downward trend (Figure 2).

\section{Comparison of Plasma $\beta$-EP Content $(\mathrm{Pg} / \mathrm{mL})$ Immediately Before Analgesia and at 120 Minutes After Analgesia}

At 120 minutes after analgesia, the content of plasma $\beta$ EP was increased in the TEAS group and decreased in 


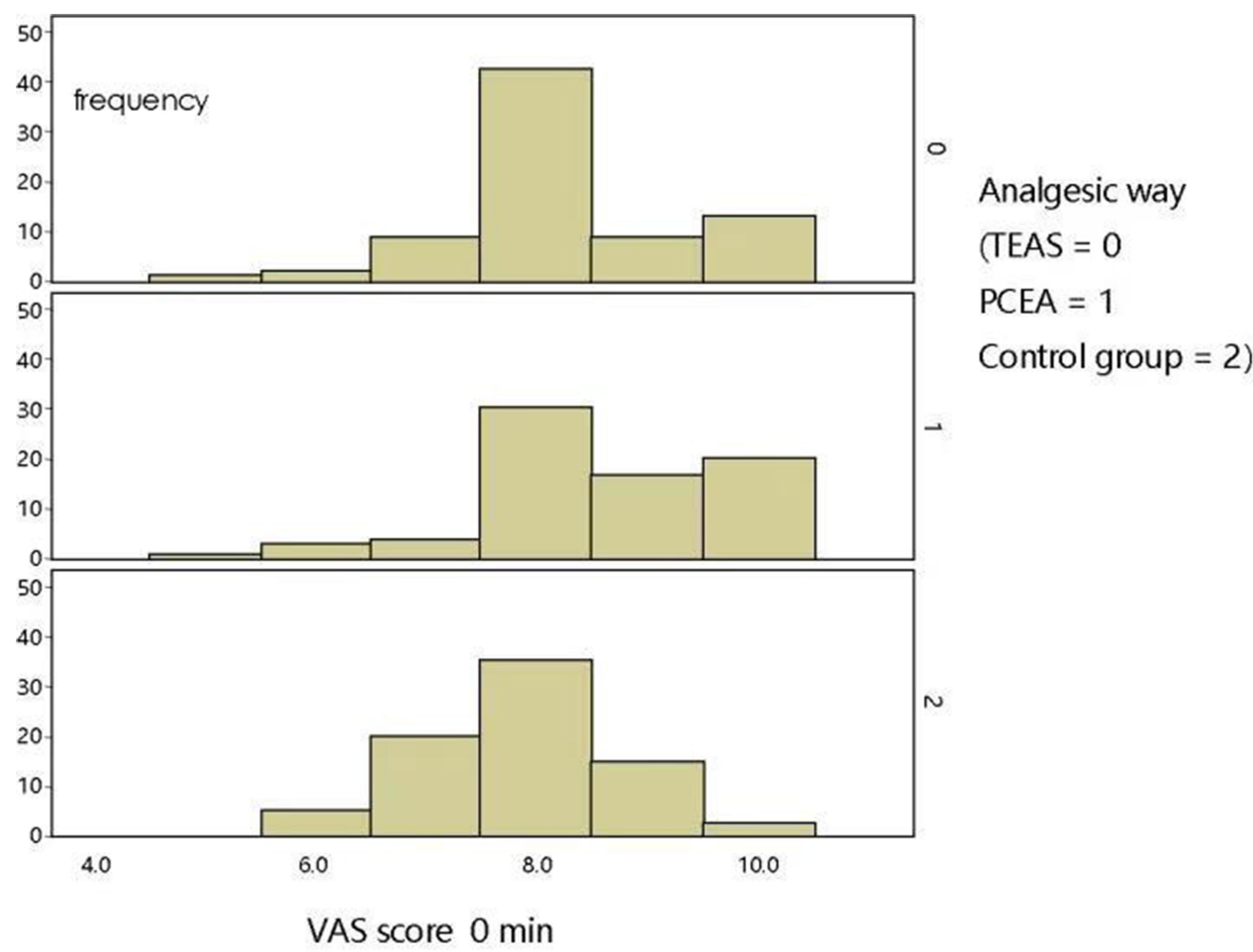

Figure I VAS score distribution of three groups before analgesia.

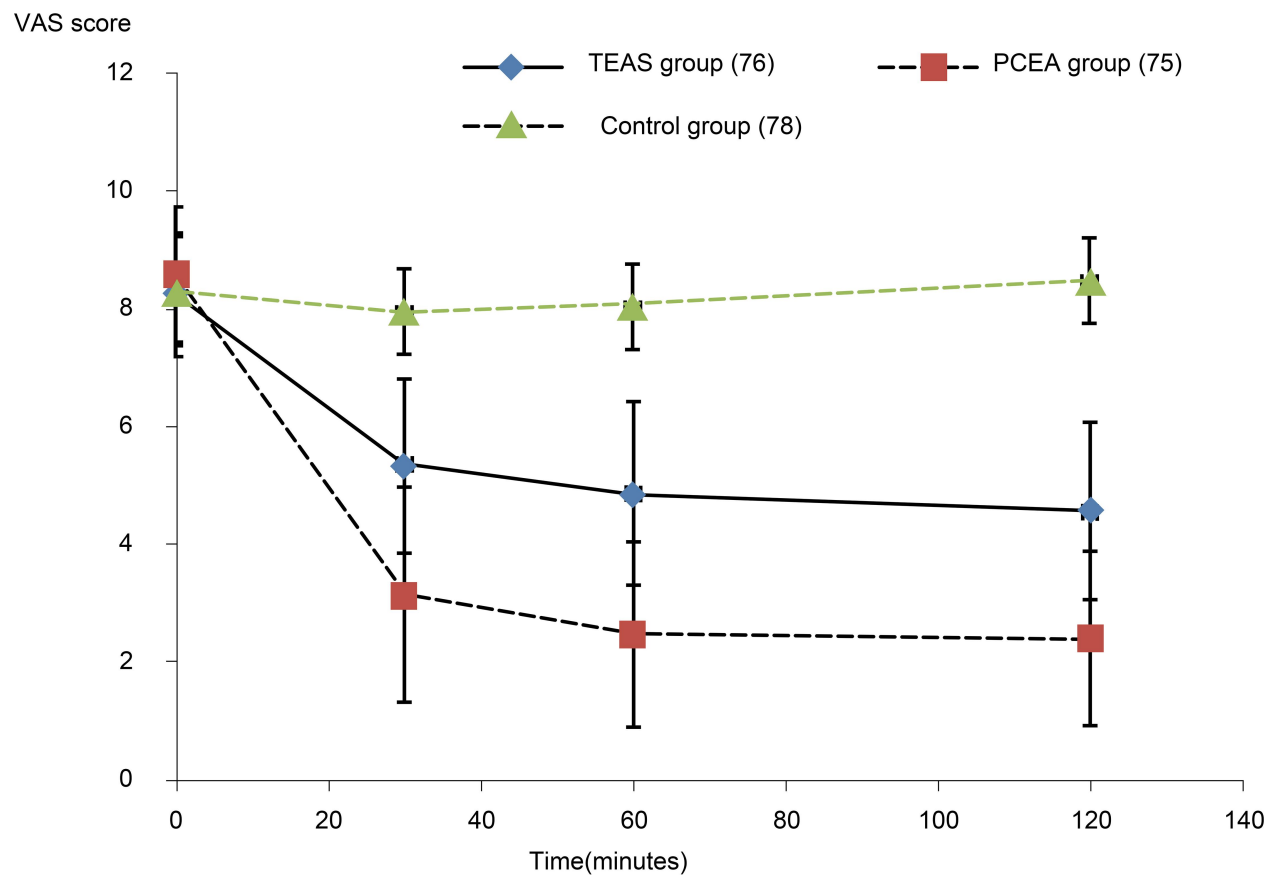

Figure 2 Comparison of VAS scores before analgesia and at each time point after analgesia: Analysis of variance of repeated measurements at different time points after analgesia among the three groups reveal that, after the implementation of analgesia, there are significant differences among different analgesia methods $\left(F_{\text {group }}=33 \mathrm{I} .26 \mathrm{I}, \mathrm{P}=\right.$ $0.000)$; the VAS score is significantly lower in the TEAS group and PCEA group than in the control group $(P=0.000 ; P=0.000)$.

the PCEA group. With the difference in $\beta$-EP content at 120 minutes after analgesia minus that immediately before analgesia as the index, there were significant differences in plasma $\beta$-EP content between the TEAS group and PCEA group, and between the TEAS group and control group $(\mathrm{P}<0.01, \mathrm{P}<0.01)$. The difference 
between the PCEA group and the control group was statistically significant $(\mathrm{P}<0.01$, Figure 3$)$.

\section{Comparison of Labor Duration of Vaginal Delivery After Analgesia}

Comparison of the duration of the first stage of labor: The duration of the first stage of labor was significantly shorter in the TEAS group and control group than in the PCEA group $(\mathrm{P}<0.01, \mathrm{P}<0.01)$. There was no significant difference in the duration of the first stage of labor between the TEAS group and the control group $(\mathrm{P}>0.05)$. Comparison of the duration of the second stage of labor: The duration of the second stage of labor was significantly shorter in the TEAS group than in the PCEA group $(\mathrm{P}<0.01)$. The duration of the second stage of labor was also shorter in the TEAS group than in the control group, but the difference was not statistically significant $(P>0.05)$. The duration of the second stage of labor was also shorter in the PCEA group than in the control group, but the difference was not statistically significant $(\mathrm{P}>0.05)$. There was no significant difference in the duration of the third stage of labor among the three groups $(P>0.05$, Figure 4$)$.

\section{Comparison of Oxytocin Use Rate and} Adverse Reaction Rate After Analgesia

The use of oxytocin during labor revealed that it was significantly lower in the TEAS group and control group than in the PCEA group $(\mathrm{P}<0.01, \mathrm{P}<0.01)$. There was no significant difference between the TEAS group and the control group $(\mathrm{P}>0.05)$. The adverse reaction rate was significantly lower in the TEAS group and the control group than in the PCEA group $(\mathrm{P}<0.01, \mathrm{P}<0.01)$. There was no significant difference between the TEAS group and the control group $(\mathrm{P}>0.05)$. In the PCEA group, 28 of 75 patients had adverse reactions. The main manifestations were different degrees of a motor block of lower limbs in seven patients, urinary retention in ten patients, nausea and vomiting in three patients, fever in five patients, and pruritus in three patients. In the TEAS group, 3 of 76 patients had adverse reactions. The main manifestations were as follows: urinary retention in two patients and fever in one patient. In the control group, 3 of 78 patients had adverse reactions. The main manifestations were urinary retention in two patients and fever in one patient (Figures 5 and 6).

\section{Comparison of the Shifting Rate to} Cesarean Section After Analgesia, the Bleeding Volume After Vaginal Delivery After Analgesia, and Neonatal Apgar Score at One Minute and Five Minutes After Analgesia

Cesarean sections were performed in all patients with indications of cesarean section during labor. There was no significant difference in the shifting rate to cesarean

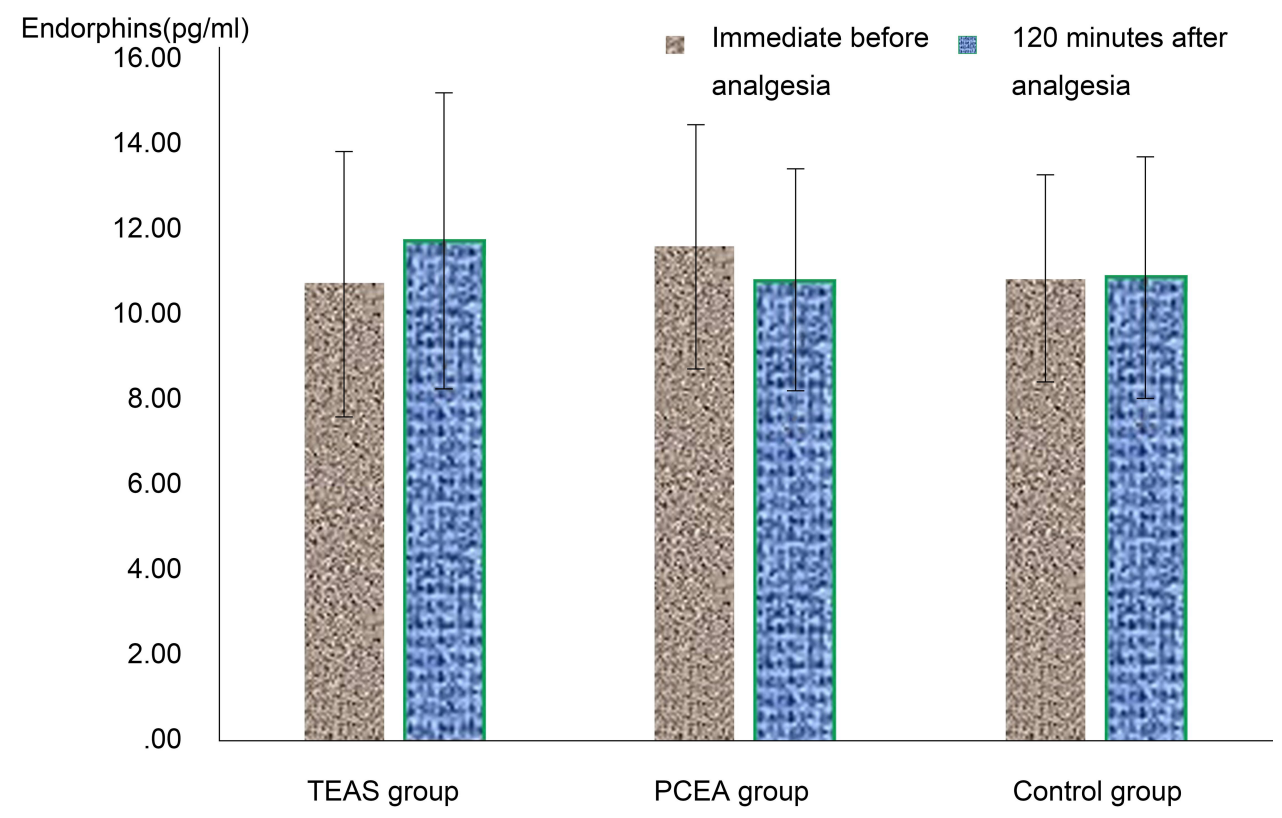

Figure 3 Comparison of $\beta$-endorphin content in three groups immediately before analgesia and at 120 minutes after analgesia: Comparison of the difference in endorphin content among the three groups at 120 minutes after analgesia and immediately before analgesia $F=13.630, P=0.000$. 


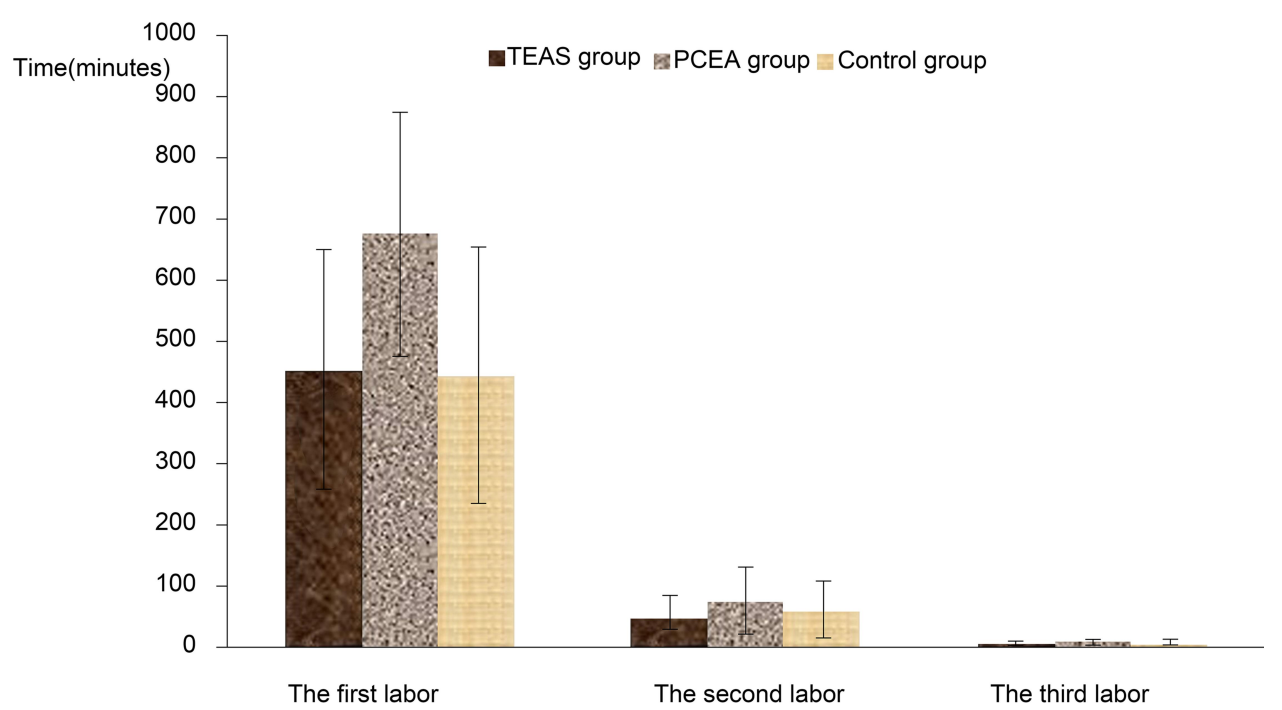

Figure 4 Comparison of the duration of of vaginal delivery among the three groups: Comparison of the duration of the first stage of labor: the duration of the first stage of labor is significantly shorter in the TEAS group and control group than in the PCEA group $(\mathrm{P}<0.0 \mathrm{I}, \mathrm{P}<0.0 \mathrm{I})$. Comparison of the duration of the second stage of labor: the duration of the second stage of labor is significantly shorter in the TEAS group than in the PCEA group $(\mathrm{P}<0.01)$.

sections among the groups $(\mathrm{P}>0.05$, Table 2$)$. There was no significant difference in postpartum bleeding volume and neonatal Apgar score at one minute and five minutes among the three groups $(\mathrm{P}>0.05$, Table 3$)$.

\section{Discussion}

This study revealed that the VAS after analgesia was significantly lower in the TEAS group and PCEA group than in the control group. The result suggests that TEAS has the same obvious analgesic effect as PCEA. Compared with the TEAS group, the PCEA group presented a larger decrease. The result suggests that the analgesic effect was better in the PCEA group than in the TEAS group. This result is consistent with that reported in the related literature. $^{7,8}$ Although the analgesic effect of the TEAS group was inferior to that of an epidural block, it could relieve labor pain to the extent that the parturient could tolerate it. In this study, the VAS of the TEAS group

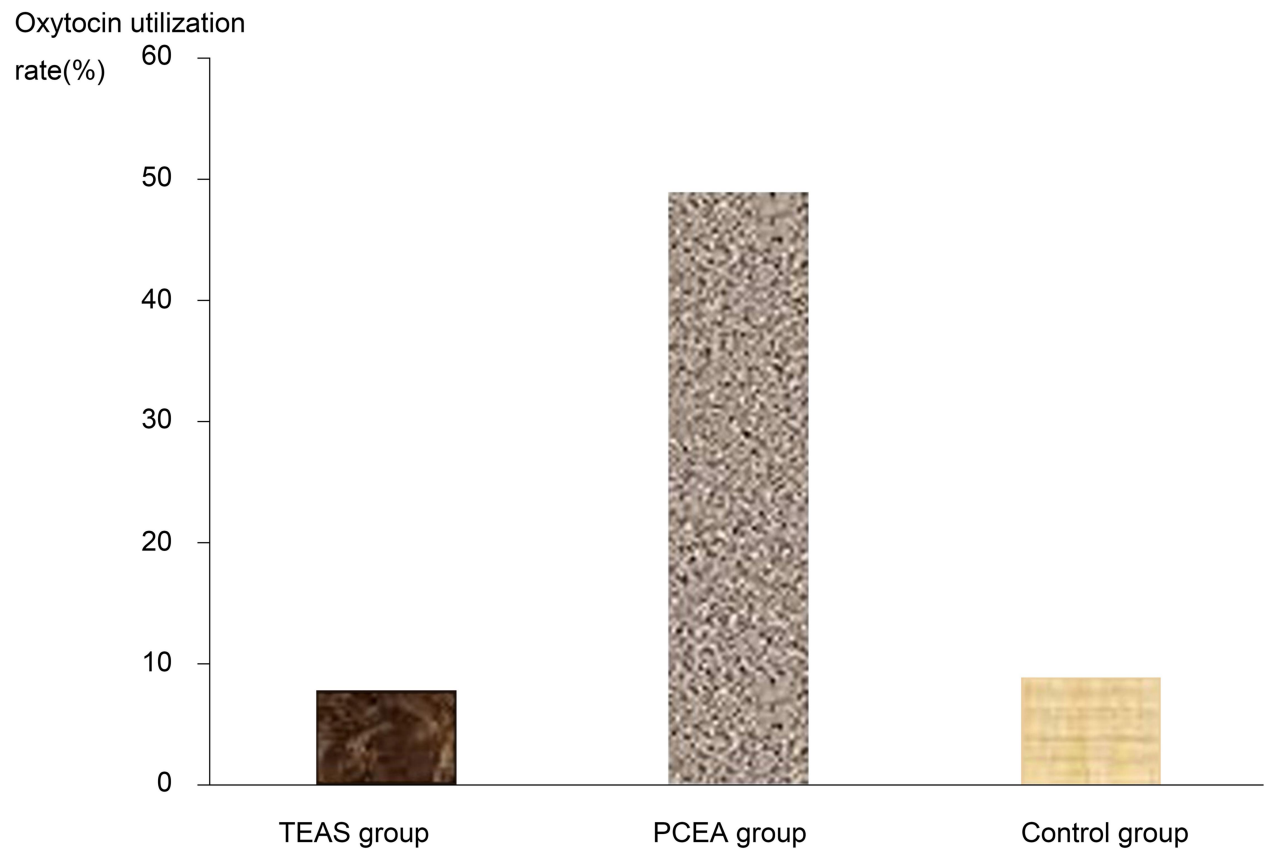

Figure 5 Comparison of oxytocin use rate of three groups after analgesia: Comparison of oxytocin use rate of three groups after analgesia: The use rate is significantly lower in the TEAS group and control group than in the PCEA group $(P=0.000, P<0.01, P=0.000, P<0.01)$. 


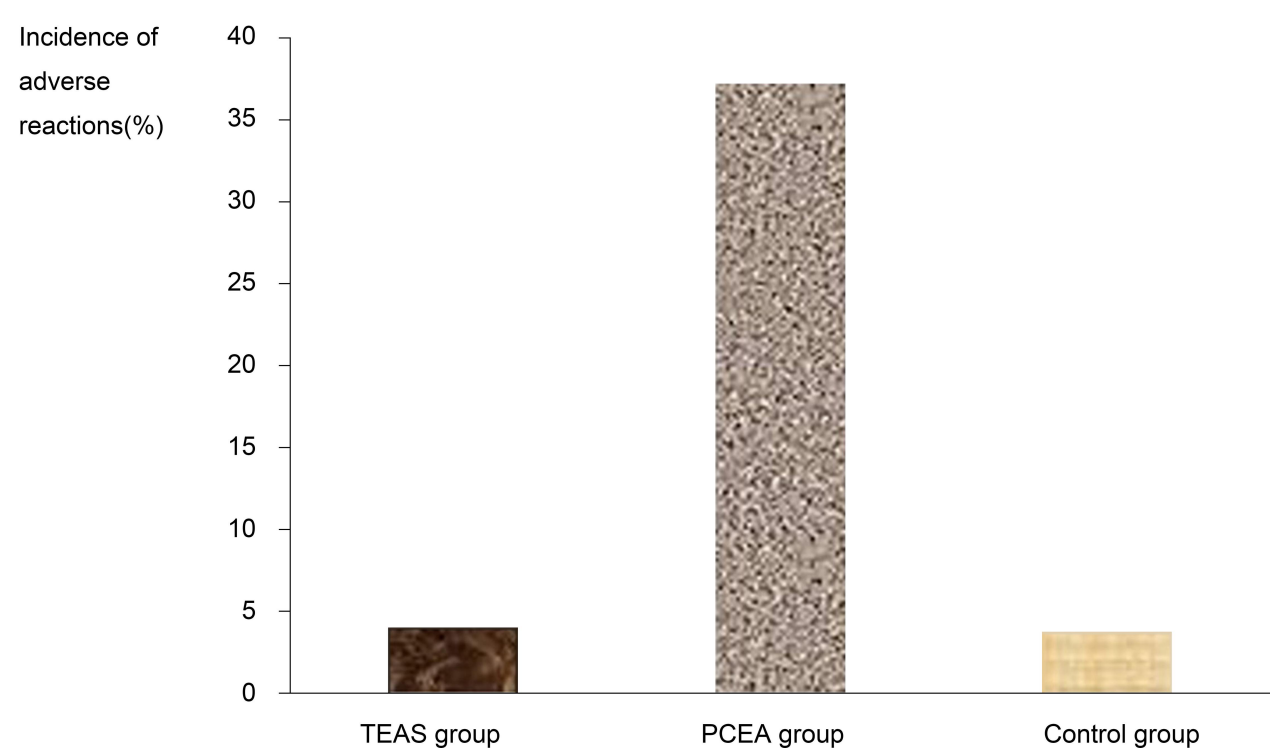

Figure 6 Comparison of the incidence of adverse reactions after analgesia among three groups: Oxytocin use rate after analgesia is significantly lower in the TEAS group and control group than in the PCEA group $(P=0.000, P<0.01, P=0.000, P<0.01)$.

decreased by more than 3 points at 60 minutes after analgesia. A comparison of analgesia at different time points revealed that the PCEA group and TEAS group presented the best analgesic effect at 60 minutes after analgesia. There was no significant change in VAS between 60-120 minutes. The result suggests that the analgesic effect of TEAS is as stable as that of PCEA. The parameters of TEAS are various. They include stimulation intensity, frequency, pulse width, duration, waveform, and frequency. Different stimulation parameters can affect the analgesic effect. When considering the individual differences of patients and the high complexity and variability of pain, the clinical application of TEAS should choose different stimulation parameters according to the pain symptoms and personality traits of different patients. It should also be adjusted timely according to the change of pain to improve the analgesic effect.

The specific analgesic mechanism of TEAS is still unclear. Traditional Chinese medicine believes that stimulating Hegu, Neiguan, Sanyinjiao, and other related

Table 2 Comparison of Cesarean Section Rate Among Three Groups of Puerperant (Cases [\%])

\begin{tabular}{|l|l|l|}
\hline Groups & Cases & Cesarean Section Rate \\
\hline TEAS group & 76 & $9(12 \%)$ \\
PCEA group & 75 & $14(19 \%)$ \\
Control group & 78 & $8(10 \%)$ \\
$\chi^{2}$ & & 2.514 \\
$P$ & & 0.284 \\
\hline
\end{tabular}

acupoints can strengthen labor contraction and relieve labor pain. ${ }^{9-11}$ In clinical application, the selected acupoints of TEAS analgesia apparatus are slightly different. ${ }^{12-14}$ In this study, Sunray fetal monitoring or neuromuscular stimulators were used, and Hegu, Neiguan, Jiaji, and Ciliao acupoints were selected. Hegu can dredge the qi and blood of meridians, acupuncture of these can strengthen labor contractions, induce labor, and labor pressure, and nourishing Hegu can treat dystocia. Traditional Chinese medicine scripture "Acupuncture and Moxibustion Mastery" also records that Neiguan can treat a variety of pain syndromes, which is the first choice for treating abdominal pain. Ciliao is an important point in gynecology. It can promote the circulation of qi, activate blood circulation, restore menstrual flow, and relieve pain. Jiaji is located on both sides of the spine and can regulate

Table 3 Comparison of the Bleeding Volume After Vaginal Delivery Under Analgesia and Neonatal Apgar Score at I Minute and 5 Minutes After Analgesia Among the Three Groups of Puerperant

\begin{tabular}{|l|c|c|c|}
\hline \multirow{2}{*}{ Group (Cases) } & \multirow{2}{*}{$\begin{array}{c}\text { The Bleeding Volume } \\
\text { After Vaginal Delivery }\end{array}$} & \multicolumn{2}{|c|}{ Apgar Score } \\
\cline { 3 - 4 } & $\mathbf{m L}$ & I Minute & $\mathbf{5}$ Minutes \\
\hline TEAS group (67) & $281.9 \pm 82.2$ & $9.75 \pm 1.02$ & $9.93 \pm 0.42$ \\
PCEA group (6I) & $319.5 \pm 223.5$ & $9.83 \pm 0.64$ & $9.93 \pm 0.41$ \\
Control=group (70) & $324.6 \pm 236.8$ & $9.81 \pm 0.60$ & $9.99 \pm 0.12$ \\
$F$ & 0.970 & 0.174 & 0.528 \\
$P$ & 0.381 & 0.840 & 0.591 \\
\hline
\end{tabular}


Yin and Yang, dredge qi and blood, and relieve pain. Modern medical theory considers that TEAS can activate small diameter noxious fibers (A $\delta$ and $\mathrm{C}$ fibers) by applying low-frequency and high-intensity acupuncture, like electrical stimulation, to induce pain descending inhibition pathways in the periaqueductal gray matter-ventromedial nucleus of the medulla oblongata-spinal cord. This can release endogenous opioid peptide neurotransmitters, such as dynorphin, endorphin, and orphanin, to participate in analgesia. The stimulation position can be acupoints, muscle tissue, and sports joints, which are located away from the site of pain. ${ }^{15-17}$ The present study revealed that the contents of plasma $\beta$-EP in the three groups changed 120 minutes after analgesia. The content of plasma $\beta$-EP increased in the TEAS group and decreased in the PCEA group. With the difference of $\beta$-EP content at 120 minutes after analgesia minus that immediately before analgesia as the index, there was a significant difference in plasma $\beta$ EP content between the TEAS group and the PCEA group, and between the TEAS group and the control group. The difference between the PCEA group and the control group was statistically significant. The result suggests that the use of TEAS may promote the production of endogenous analgesic substance $\beta$-EP and participate in analgesia. PCEA has an analgesic effect through the combination of exogenous opioid drugs to opioid receptors in the body. It can reduce the stress response accordingly by reducing the production of endogenous analgesic substances, such as $\beta$ EP, appropriately. ${ }^{7}$

In the present study, after eliminating cesarean sections, the duration of labor of vaginal delivery was compared among the three groups. Comparison of the duration of the first stage of labor: The duration of the first stage of labor was significantly shorter in the TEAS group and control group than in the PCEA group. There was no significant difference in the duration of the first stage of labor between the TEAS group and the control group. The result suggests that PCEA can prolong the first stage of labor. Comparison of the duration of the second stage of labor: The duration of the second stage of labor was significantly shorter in the TEAS group than in the PCEA group. The difference was highly significant statistically. The duration of the second stage of labor was also shorter in the TEAS group than in the control group, but differences were not statistically significant. The duration of the second stage of labor was also shorter in the PCEA group than in the control group, but the difference was not statistically significant. These results suggest that TEAS can accelerate the second stage of labor, and PCEA can prolong the first stage of labor. The mechanism may be that TEAS can enhance labor contraction by stimulating Hegu to accelerate the progress of the labor process. PCEA delays the progress of labor by inhibiting the labor contraction through sympathetic regulation and reducing the release of endogenous oxytocin. There was no significant difference in the duration of the third stage of labor among the three groups. Yin et $\mathrm{al}^{15}$ revealed that although PCEA prolonged the second stage of labor and total stages of labor, it did not affect the outcome of mother and infant. But Han et $\mathrm{al}^{16}$ reported that continuous PCEA had no effect on the first and second stages of labor but increased oxytocin use.

In this study, uterine atony occurred during labor. If, after general treatment such as rest, diet, and activity, the patient still presented coordinated uterine atony, and fetal heart rate was good, the fetal position was normal, and the head and basin were matched, the patient was treated with small doses of oxytocin to strengthen labor contraction. In this study, a comparison of oxytocin use rate revealed that it was significantly higher in the PCEA group than in the TEAS group and control group. The reason may be that TEAS can strengthen labor contraction by stimulating Hegu, and PCEA reduces the release of endogenous oxytocin by inhibiting regulation of the sympathetic nerve on labor contraction, causing a reduction of the intensity of labor contractions and prolongation of the interval time. ${ }^{19}$ Therefore, the oxytocin use rate was much higher in the PCEA group than in the TEAS group or control group. Adverse reaction rates were significantly higher in the PCEA group than in the TEAS group or control group. The result suggests that PCEA can significantly increase the incidence of adverse reactions. In this study, there were three cases of adverse reactions in the TEAS group and three in the control group. Urine retention was induced by premature rupture of membranes. Bed rest time was longer, and fever was caused by the infection of premature rupture of membranes. In this study, in the PCEA group, 28 of 75 patients had adverse reactions. The main manifestations were different degrees of lower limb motor blocks, urinary retention, nausea and vomiting, fever, and itching. On analysis, it was found that the high incidence of side effects of PCEA of labor analgesia may be related to the type, dosage, timing, anesthesia level, and block range of analgesic drugs. Several reports ${ }^{12,13}$ revealed that women with PCEA increased the use of oxytocin and prolonged the second stage of labor, and had motor 
blocks, fever, and urinary retention. This was consistent with this study.

In this study, a cesarean section was performed on all patients with indications of cesarean section during labor. Comparisons revealed that there was no difference in the shifting rate to a cesarean section among the three groups after analgesia. In this study, the three groups did not implement instrumental midwifery, but shifting to a cesarean section occurred in 14 patients in the PCEA group, nine patients in the TEAS group, and eight patients in the control group, respectively. Although the difference between the three groups was not statistically significant, the rate of cesarean section increased in the PCEA group. Whether PCEA affects cesarean section rate and instrumental delivery rate is expected to be confirmed by a larger sample study.

In this study, there were no differences in the bleeding volume after vaginal delivery after analgesia or neonatal Apgar score at one minute and five minutes after analgesia among the three groups. The result suggests that there was no obvious adverse effect on maternal and neonatal obstetric outcomes. This was consistent with the results reported in related studies. ${ }^{7,15-19}$

\section{Conclusion}

Although the analgesic effect of TEAS on labor is not ideal, its advantages of being non-invasive, non-drug, economical, easy to operate, and no obvious adverse reactions for mother and child, has attracted more and more attention. Modern labor analgesia should consider not only the analgesic effect but also the safety of mother and baby. In clinical work, a variety of non-drug labor analgesia methods will be combined to complement each other. TEAS labor analgesia instrument and doula can be used or assisted by respiratory analgesia to improve the analgesic effect of TEAS and promote natural childbirth.

\section{Ethics Approval and Consent to Participate}

I confirm that I have read the Editorial Policy pages. This study was conducted with approval from the Ethics Committee of The Affiliated Hospital of Qingdao University. This study was conducted in accordance with the declaration of Helsinki. Written informed consent was obtained from all participants.

\section{Consent for Publication}

All participants signed a document of informed consent.

\section{Acknowledgments}

We would like to acknowledge the hard and dedicated work of all the staff that implemented the intervention and evaluation components of the study.

\section{Funding}

There is no funding to report.

\section{Disclosure}

The authors declare that they have no conflicts of interest for this work.

\section{References}

1. Kisuule F, Howell E. Hospital medicine beyond the United States. Int J Gen Med. 2018;11:65-71. doi:10.2147/IJGM.S151275

2. Tzeng YL, Yang YL, Kuo PC, Lin YC, Chen SL. Pain, anxiety, and fatigue during labor: a prospective, repeated measures study. $J$ Nurs Res. 2017;25(1):59-67. doi:10.1097/jnr.0000000000000165

3. Lim G, Farrell LM, Facco FL, Gold MS, Wasan AD. Labor analgesia as a predictor for reduced postpartum depression scores: a retrospective observational study. Anesth Analg. 2018;126 (5):1598-1605. doi:10.1213/ANE.0000000000002720

4. Chen L. The influence of high quality accompanying childbirth on the outcome of childbirth. Qinghai Med J. 2017;47(09):46-48.

5. Shaaban OM, Abbas AM, Mohamed RA, Hafiz H. Lack of pain relief during labor is blamable for the increase in the women demands towards cesarean delivery: a cross-sectional study. Facts Views Vis Obgyn. 2017;9(4):175-180.

6. Smith LA, Burns E, Cuthbert A. Parenteral opioids for maternal pain management in labour. Cochrane Database Syst Rev. 2018;6: CD007396.

7. Anim-Somuah M, Smyth RM, Cyna AM, Cuthbert A. Epidural versus non-epidural or no analgesia for pain management in labour. Cochrane Database Syst Rev. 2018;5(5):CD000331. doi:10.1002/ 14651858.CD000331.pub4

8. Czech I, Fuchs P, Fuchs A, et al. Pharmacological and non-pharmacological methods of labour pain relief-establishment of effectiveness and comparison. Int $J$ Environ Res Public Health. 2018;15(12):2792. doi:10.3390/ijerph15122792

9. Grant EN, Tao W, Craig M, McIntire D, Leveno K. Neuraxial analgesia effects on labour progression: facts, fallacies, uncertainties and the future. BJOG. 2015;122(3):288-93. doi:10.1111/14710528.12966

10. Qi F, Huang SQ, Ding Y. Investigation and analysis of labor analgesia service in China. Maternal Child Health Care China. 2019;34 (09):1937-1941.

11. Sng BL, Sia ATH. Maintenance of epidural labour analgesia: the old, the new and the future. Best Pract Res Clin Anaesthesiol. 2017;31 (1):15-22. doi:10.1016/j.bpa.2017.01.002

12. Sun YL, Yang XL, Zhu Y, Long Q. Effect of the Doula instrument muscle electrical stimulation and intravertebral anesthesia on the delivery outcome. Maternal Child Health Care China. 2018;33 (21):4987-4990.

13. Lei WP, Huang YQ, Xiao C, Sun JL. Clinical observation of epidural anesthesia assisted by electric stimulation of percutaneous acupoint for labor analgesia.Modern. Pract Med. 2019;31(04):527-529.

14. Zhu J. Influence of acupoint massage on analgesia effect and labor process of primipara. Electron J Pract Gynecol Endocrinol. 2019;6 (31):149. 
15. Yin H, Hu R. A cohort study of the impact of epidural analgesia on maternal and neonatal outcomes. J Obstet Gynaecol Res. 2019;45 (8):1435-1441. doi:10.1111/jog. 13988

16. Han B, Xu M. A comprehensive analysis of continuous epidural analgesia's effect on labor and neonates in maternal hypertensive disorder patients. Pregnancy Hypertens. 2017;7:33-38. doi:10.1016/ j.preghy.2016.12.003

17. Naito Y, Ida M, Yamamoto R, Tachibana K, Kinouchi K. The effect of labor epidural analgesia on labor, delivery, and neonatal outcomes: a propensity score-matched analysis in a single Japanese institute. $J A$ Clin Rep. 2019;5(1):40. doi:10.1186/s40981-019-0260-z
18. Penuela I, Isasi-Nebreda P, Almeida H, López M, Gomez-Sanchez E, Tamayo E. Epidural analgesia and its implications in the maternal health in a low parity comunity. BMC Pregnancy Childbirth. 2019;19 (1):52. doi:10.1186/s12884-019-2191-0

19. Herrera-Gómez A, De Luna-bertos E, Ramos-Torrecillas J, Ocaña-Peinado FM, Ruiz C, García-Martínez O. Risk assessments of epidural analgesia during labor and delivery. Clin Nurs Res. 2018;27(7):841-852. doi:10.1177/1054773817722689

\section{Publish your work in this journal}

The International Journal of General Medicine is an international, peer-reviewed open-access journal that focuses on general and internal medicine, pathogenesis, epidemiology, diagnosis, monitoring and treatment protocols. The journal is characterized by the rapid reporting of reviews, original research and clinical studies across all disease areas. The manuscript management system is completely online and includes a very quick and fair peer-review system, which is all easy to use. Visit http://www.dovepress.com/ testimonials.php to read real quotes from published authors.

Submit your manuscript here: https://www.dovepress.com/international-journal-of-general-medicine-journal 